\title{
Hyaluronic acid hydrogels incorporating platelet lysate enhance human pulp cell proliferation and differentiation
}

\author{
Leopoldina D. F. Almeida ${ }^{1,2,3,4} \cdot$ Pedro S. Babo ${ }^{3,4} \cdot$ Cristiana R. Silva $^{3,4}$ - Márcia T. Rodrigues ${ }^{3,4}$ - Josimeri Hebling ${ }^{2}$. \\ Rui L. Reis ${ }^{3,4,5} \cdot$ Manuela E. Gomes ${ }^{3,4,5}$
}

Received: 16 December 2017 / Accepted: 14 May 2018 / Published online: 14 June 2018

(c) Springer Science+Business Media, LLC, part of Springer Nature 2018

\begin{abstract}
The restoration of dentine-pulp complex remains a challenge for dentists; nonetheless, it has been poorly addressed. An ideal system should modulate the host response, as well as enable the recruitment, proliferation and differentiation of relevant progenitor cells. Herein was proposed a photocrosslinkable hydrogel system based on hyaluronic acid (HA) and platelet lysate (PL). PL is a cocktail of growth factors (GFs) and cytokines involved in wound healing orchestration, obtained by the cryogenic processing of platelet concentrates, and was expected to provide the HA hydrogels specific biochemical cues to enhance pulp cells' recruitment, proliferation and differentiation. Stable HA hydrogels incorporating PL (HAPL) were prepared after photocrosslinking of methacrylated HA (Met-HA) previously dissolved in PL, triggered by the Ultra Violet activated photoinitiator Irgacure 2959. Both the HAPL and plain HA hydrogels were shown to be able to recruit cells from a cell monolayer of human dental pulp stem cells (hDPSCs) isolated from permanent teeth. The hDPCs were also seeded directly over the hydrogels $\left(5 \times 10^{4}\right.$ cells/hydrogel $)$ and cultured in osteogenic conditions. Cell metabolism and DNA quantification were higher, in all time-points, for PL supplemented hydrogels $(p<0,05)$. Alkaline phosphatase (ALPL) activity and calcium quantification peaks were observed for the HAPL group at 21 days $(p<0,05)$. The gene expression for $A L P L$ and COLIAl was up-regulated at 21 days to HAPL, compared with HA group $(p<0,05)$. Within the same time point, the gene expression for RUNX2 did not differ between the groups. Overall, data demonstrated that the HA hydrogels incorporating PL increased the cellular metabolism and stimulate the mineralized matrix deposition by hDPSCs, providing clear evidence of the potential of the proposed system for the repair of damaged pulp/dentin tissue and endodontics regeneration.
\end{abstract}

These authors contributed equally: Leopoldina F. D. Almeida, Pedro S. Babo.

$\triangle$ Manuela E. Gomes

megomes@dep.uminho.pt

1 Department of Clinical and Social Dentistry, Federal University of Paraíba, João Pessoa, PB, Brazil

2 Department of Orthodontics and Pediatric Dentistry, Araraquara Dental School, State of São Paulo University, Araraquara, SP, Brazil

3 3B's Research Group, I3Bs-Research Institute on Biomaterials, Biodegradables and Biomimetics, University of Minho,

\section{Introduction}

Endodontic regenerative approaches have explored the stimulatory effect of synthetic and biologic origin biopolymers aiming the regeneration of injured dentin-pulp complex [1]. These therapies explore the endogenous regenerative potential of the endodontic tissues and procure to enhance it by combining biopolymers with adequate biochemical

Headquarters of the European Institute of Excellence on Tissue Engineering and Regenerative Medicine, AvePark, Parque de Ciência e Tecnologia, Zona Industrial da Gandra, 4805-017 Barco, Guimarães, Portugal

4 ICVS/3B's-PT Government Associate Laboratory, Braga/ Guimarães 4805-017, Portugal

5 The Discoveries Centre for Regenerative and Precision Medicine, Headquarters at University of Minho, 4805-017 Barco,

Guimarães, Portugal 
stimuli [1]. Among the most reported biopolymers for endodontic therapy are hyaluronic acid (HA) [2-4], chitosan [5], alginate [6], gelatin [7-9], collagen [10, 11], fibrin [12], and silk fibroin [13]. These natural-origin molecules resemble the extracellular matrix (ECM) components, providing unique properties to the specific regenerative approach, thereby enhancing their biocompatibility and biological recognition.

HA is a glycosaminoglycan, composed by units of Dglucuronic acid and N-acetyl-D-glucosamine acid [14], and is present in the central region of pulp were the undifferentiated cells are located [15]. HA suitability for regenerative medicine purposes relates with its biocompatibility and ability to be modified through both its carboxyl $[16,17]$ and hydroxyl groups $[18,19]$ to produce stable hydrogels. It has been shown to be suitable for endodontic regenerative procedures, displaying immunomodulatory, and proangiogenic features [4]. Moreover, the methacrylation of HA on its hydroxyl moieties, allows the production of injectiqable photocrosslinkable HA hydrogels [18, 20]. These injectable hydrogels are ideal for endodontic regenerative approaches as they can easily penetrate throughout the root canal system and crosslink upon specific light activation. Nevertheless, the HA hydrogels lack specific biochemical cues to orchestrate tissue regeneration.

In this study, we investigated the potential of platelet lysate (PL) to provide HA hydrogels with a favorable microenvironment for pulp cell recruitment, proliferation, and differentiation. PL is a rich cocktail of growth factors, cytokines, and clot-forming proteins involved in the wound healing process $[21,22]$. It is obtained by the simple disruption of the membranes of platelets contained in platelet concentrates by freeze-drying cycles and has been proposed to be more effective and reliable in comparison with the PRHds activated with thrombin or calcium (e.g., PRP) [22, 23]. The incorporation of PL into HA hydrogels has been shown to enhance the adhesion and proliferation of human adipose tissue derived cells (hASCs), and periodontal ligament fibroblasts $[18,19]$. Among the GFs present in PL are the FGF, VEGF, PDGF, TGF- $\beta 1$, TGF- $\beta 2$, and IGF $[21,22]$ which are known to promote the formation of tertiary dentin and dental pulp repair [24]. Moreover, the chemotactic and proangiogenic GFs present in PL (PDGF and VEGF) [25], can facilitate the recruitment of progenitor cells from the amputated pulp tissues. Additionally, the antimicrobial properties exhibited by PL $[18,26]$ might reduce the recurrence of endodontic diseases. However, the effect of human PL associated with biomaterials has not yet been evaluated in human dental pulp cell cultures.

These pulp resident cells have a high proliferation rate and pluripotent characteristics [27], making them suitable for testing materials for endodontic regenerative therapies $[5,10]$. Therefore, this study evaluated the hypothesis that a
HA-based photopolymerizable hydrogel associated with human PL would promote the recruitment, increase the proliferation and metabolism and induce the differentiation in primary cultures isolated from human dental pulp.

\section{Material and Methods}

\subsection{Ethics statement}

The collection and manipulation of human samples, namely teeth and platelet concentrate batches, were performed in accordance with the international guidelines. Human teeth were extracted for orthodontic reasons at Service of Oral Surgery of the Malo Clinic Dental Care (Portugal), following the standards of quality and security (as established in 2004/23/CE, 2006/17/CE, and 2006/86/CE), under an institutional board protocol established and approved by the ethical commission for health at 23/12/2014. All human donors provided a written informed consent before enrolment in accordance with the Declaration of Helsinki (59th WMA General Assembly, Seoul, Korea, October 2008).

Platelet components from whole blood or apheresis for the production of the PL batches were collected from adult, healthy, volunteer, regular blood donors compliant with the requisites stablished in annex II and III of directive 2004/33/ CE and annex IV of directive 2002/98/CE. The collections were performed at Serviço de Imunohemoterapia-CHSJ (Porto, Portugal) provided under an approved institutional board protocol. All the donations were anonymous in agreement with the European data protection directive 95/ 46/EC.

\subsection{Production of methacrylated hyaluronic acid}

High molecular weight (1.5-1.8 MDa) hyaluronic acid (HA; S. equii, Sigma-Aldrich, Missouri, USA) was methacrylated following a previously stablished protocol [18, 19, 28]. In brief, $1 \%$ (w/v) HA solution (in distilled water) was reacted with methacrylic anhydride (10 times molar excess) at $\mathrm{pH}$ 8.0-8.5, adjusted with $\mathrm{NaOH} 5 \mathrm{M}$ (Sigma-Aldrich, Missouri, USA), at $4{ }^{\circ} \mathrm{C}$. The reaction was conducted for $16 \mathrm{~h}$, under constant agitation, and subsequently the hyaluronic acid was precipitated with ethanol $\left(-20^{\circ} \mathrm{C}\right)$. Afterwards the precipitated material was centrifuged at $6000 \times g$ (3 times), and then submitted to dialysis against mili-Q water for 7 days. Finally, the Met-HA solution was filtered, freeze-dried for 7 days, and subsequently sterilized with ethylene oxide.

The methacrylation degree, defined as the percentage of methacrylated disaccharides, was further determined to be $10.9 \pm 1.07 \%$ by analysis of the Met-HA ${ }^{1} \mathrm{H}-\mathrm{NMR}$ spectra (Fig. 1a). 
Fig. 1 Hyaluronic acid photocrosslinkable hydrogels. a ${ }^{1} \mathrm{H}-\mathrm{NMR}$ spectrum of the methacrylated hyaluronic acid (Met-HA). b Illustrative cylindrical-shaped $(5 \mathrm{~mm}$ diameter $\times 5 \mathrm{~mm}$ thick) HA hydrogels plain (HA) or incorporating platelet lysate (HAPL) a

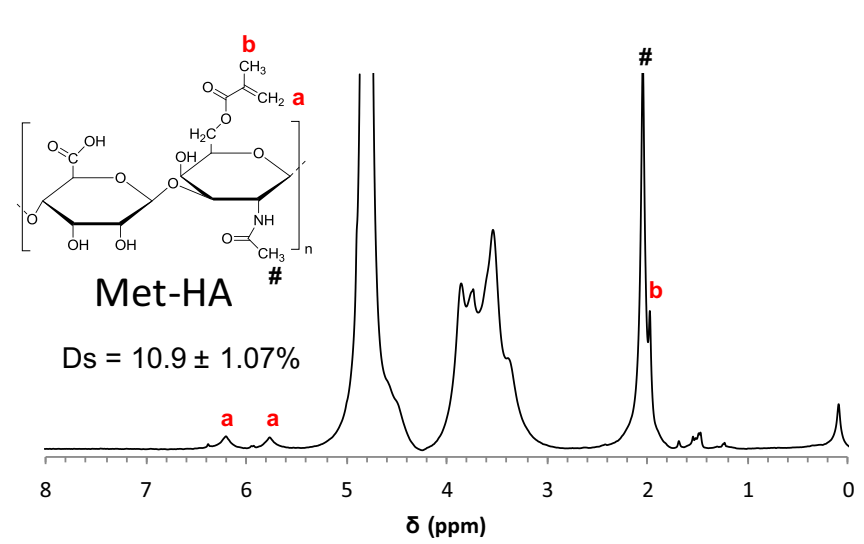

b
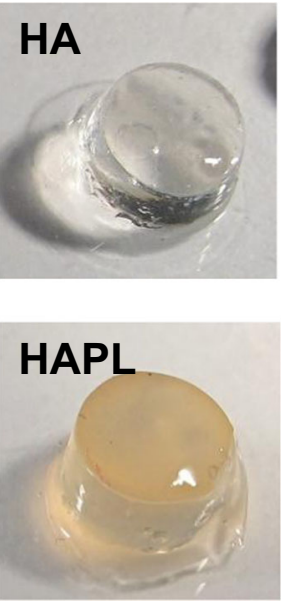

\subsection{Platelet lysate}

Platelet lysate (PL) was produced by cryogenic processing of outdated ( 5 days old) platelet concentrates (PC) produced by plasmapheresis, as previously described [18, 21, 22]. In brief, twelve PC ( $10^{6}$ platelets/ $\left.\mu \mathrm{L}\right)$ batches were randomly selected and pooled. Then, the platelets' membrane was disrupted by performing 3 consecutive freezing and melting cycles at $37{ }^{\circ} \mathrm{C}$ and $-80{ }^{\circ} \mathrm{C}$. After this process, the PL pool was aliquoted in sterile Falcon tubes $(15 \mathrm{~mL})$ and stored at $-80{ }^{\circ} \mathrm{C}$ until further use. Just before use, the PL was thaw at room temperature, centrifuged $(1400 \times g$ for $2 \mathrm{~min})$ and filtered $(0.45 \mu \mathrm{m})$ to remove any cell debris or clots.

\subsection{Production of photocrosslinkable hyaluronic acid hydrogels}

Plain hyaluronic acid hydrogels (HA-control group) or HA hydrogels incorporating $100 \%$ (v/v) PL (HAPL-experimental group) were produced as described elsewhere[18]. In order to produce the photocrosslinkable hydrogel formulations, $0.1 \%(\mathrm{w} / \mathrm{v})$ solutions of the photoinitiator Irgacure 2959 (2-Hydroxy-4'-(2-hydroxyethoxy)-2methylpropiophenone; Sigma-Aldrich, Missouri, USA), were produced both in PBS and PL. Then, Met-HA was dissolved at a concentration of $1.5 \%(\mathrm{w} / \mathrm{v})$ in both of the PBS or PL photoinitiator solutions. Given the high viscosity of the Met-HA solutions, these were subjected to centrifugation and vortexing cycles every 30 minutes, protected from the light, to assure their proper homogenization.

Afterwards, $75 \mu \mathrm{L}$ of the previously prepared solutions were injected into disk-shaped PDMS molds $(9 \mathrm{~mm}$ diameter $\times 1 \mathrm{~mm}$ thick) by means of a pipette for viscous solutions (Gilson, Middleton, WI). Subsequently the samples were light polymerized by exposure to UV light ( $\lambda$ $320 \mathrm{~nm}$ ) (Omnicure series 2000 EXFO S2000-XLA) with a power of $800 \mathrm{~mW}$ and exposure time of 70 seconds, rendering stable disk-shaped hydrogels.

\subsection{Isolation and culture of human dental pulp stem cells}

Human dental pulp stem cells (DPCS) were isolated from sound human permanent molars $(n=3$; Fig. $2 \mathrm{a}$ ), removed by orthodontic reasons, as previously described [29]. Briefly, the pulp tissue was digested with collagenase $(3 \mathrm{mg} / \mathrm{mL})$ for $2 \mathrm{~h}$, at $37^{\circ} \mathrm{C}$, the cells were seeded in polystyrene culture plates and cultivated in DMEM-F12 (Gibco, Grand Island, NY, USA) supplemented with $10 \%$ fetal bovine serum (FBS; Gibco, Grand Island, NY, USA) and $1 \%$ antibiotic/antimycotic solution (Alfagene, Life Technologies, Scotland), hereby designated as basal medium. Each 2-3 days the basal medium was replaced and the cells cultured until $80 \%$ confluence, before being subcultured. Within this study were used cells between the third and eighth passages.

\subsection{Human DPCs characterization by flow cytometry analysis}

The hDPCs populations were further characterized by flow cytometry. Initially, the cells were cultured with basal medium until sub-confluence in $175 \mathrm{~mm}^{2}$ flasks. After, the cells at passage 3 were detached and counted in order to reach a cell density of 150,000 cells/tube. The cellcontaining tubes were centrifuged at $200 \times g$ during $7 \mathrm{~min}$. Then, cells were incubated for $30 \mathrm{~min}$ at room temperature with mouse anti-human antibodies, namely CD73PE, 

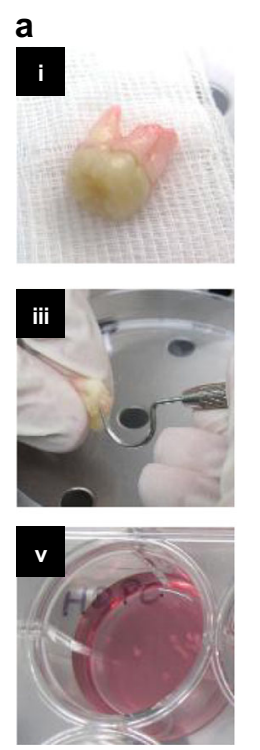

b

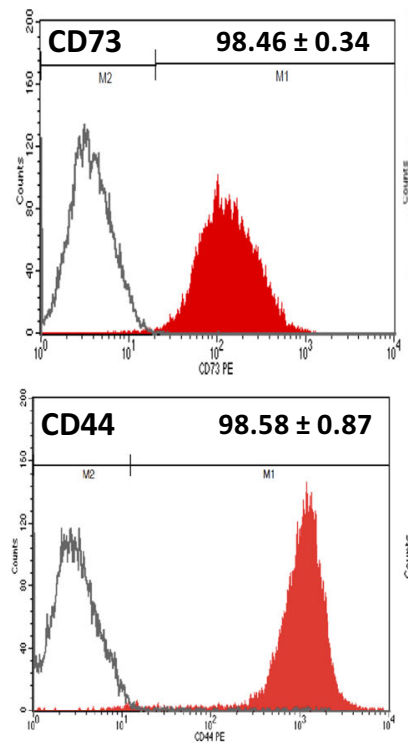

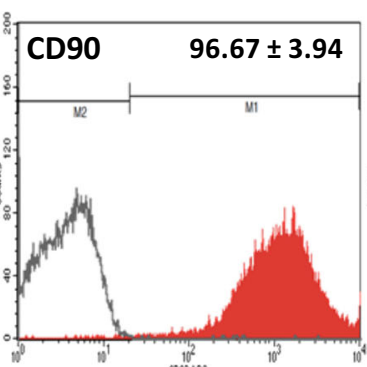
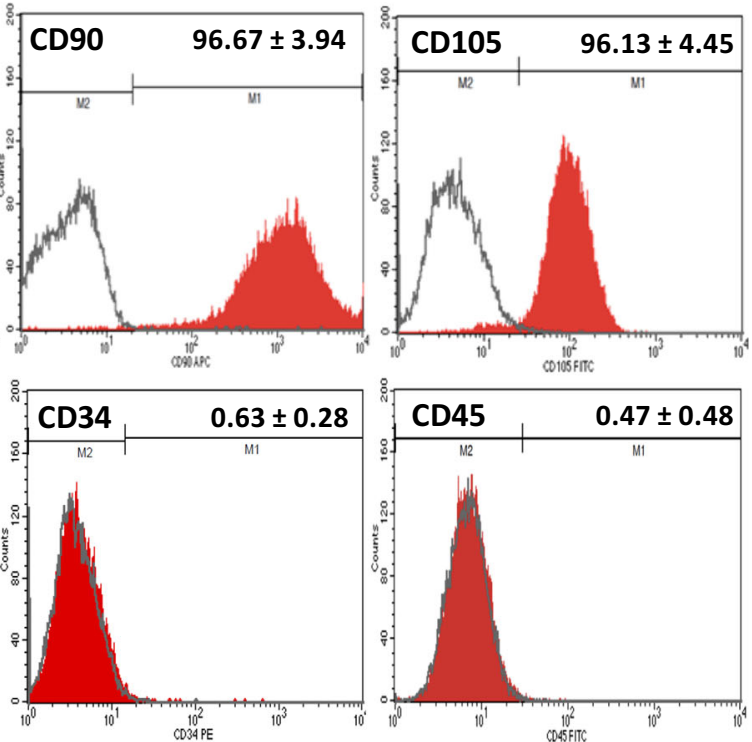

Fig. 2 Human dental pulp cells (hDPCs). a Isolation of hDPCs from (i) sound permanent teeth started by the (ii) separation of teeth crown and root, in order to expose the pulp tissue. The pulp tissue was then (iii) removed from the pulp chamber, (iv) minced, and (v) enzymatically digested to release the cell from the extracellular matrix. The digested tissue was cultured in polystyrene tissue culture plates, to which the hDPCs (vi) adhered assuming a spindle-like morphology. b Flow cytometry analysis of hDPCs populations isolated from dental pulp tissue for CD73, CD90, CD105, CD44, CD34, and CD45 expression

in culture for the time intervals of 7,14 , and 21 days, with the osteogenic medium being changed every $72 \mathrm{~h}$. Two independent assays were performed for each of two hDPCs isolated populations ( $n=16 /$ group). The metabolic activity and the proliferation of hDPCs and expression of osteogenic markers were determined after 7,14 , and 21 days of culture.

\subsubsection{Cell metabolic activity}

Cell metabolic activity was determined by means of the Alamar Blue Assay ( $n=16$ /group) (Thermo Fisher Scientific) as by manufacturer's recommendation. At each time point, the samples were transferred to a new tissue culture plate and incubated in $1 \mathrm{~mL}$ of $10 \%(\mathrm{v} / \mathrm{v})$ solution of Alamar blue reagent in basal medium at $37^{\circ} \mathrm{C}$ for $3 \mathrm{~h}$. After this, $200 \mu \mathrm{L}$ of supernatant were distributed (in triplicate) in 96-well plates, and the fluorescence recorded in a fluorescence multiplate reader (Synergy H1 Hybrid Reader, Biotek, Vermont, USA) (excitation: $530 \mathrm{~nm} / \mathrm{emission}$ : 590). After this, the samples were washed with PBS solution and inserted in Eppendorf flasks containing $1.0 \mathrm{~mL}$ of ultrapure water, and stored at $-80^{\circ} \mathrm{C}$ for further dsDNA quantification.

\subsubsection{Cell proliferation}

The cell proliferation was determined as a function of the dsDNA amount over time ( $n=16 /$ group). Cell lysis was performed by sonicating the samples for $30 \mathrm{mins}$ and the 
Table 1 List of primers used in this study

\begin{tabular}{llll}
\hline Gene & NCBI ref & T $\left({ }^{\circ} \mathbf{C}\right)$ & Primer sequence \\
\hline ALPL & NM_000478.4 & 59 & $\begin{array}{l}\text { Forward: 5' GAAGGAAAAGCCAAGCAGGC 3' } \\
\text { Reverse: 5' GGGGGCCAGACCAAAGATAG 3' }\end{array}$ \\
COL1A1 & NM_000088.3 & 60 & $\begin{array}{l}\text { Forward: 5' CCCCAGCCACAAAGAGTCTAC 3' } \\
\text { Reverse: 5' TTGGTGGGATGTCTTCGTCT 3' }\end{array}$ \\
RUNX2 & NM_001024630.3 & 60 & $\begin{array}{l}\text { Forward: 5' CCGGAATGCCTCTGCTGTTA 3' } \\
\text { Reverse: 5' TGTCTGTGCCTTCTGGGTTC3' }\end{array}$ \\
18S (housekeeping) & NR_046235.3 & 59 & $\begin{array}{l}\text { Forward: 5' GAAACCTTCCGACCCCTCTC 3' } \\
\text { Reverse: 5' TACGAGGTCGATTTGGCGAG 3' }\end{array}$ \\
\hline
\end{tabular}

dsDNA was quantified by the Pico Green assay (QuantiT $^{\mathrm{TM}}$ PicoGreen ${ }^{\circledR} \mathrm{dsDNAreagent,} \mathrm{Invitrogen,} \mathrm{Life} \mathrm{Technol-}$ ogies, Scotland), as by manufacturer's instructions.

\subsubsection{Alkaline phosphatase activity}

Alkaline phosphatase (ALPL) activity was measured by the specific conversion of p-nitrophenyl phosphate (pNPP) (Sigma, St. Louis, MO) into p-nitrophenol (PNP) $(n=16 /$ group). For this purpose, the cell lysate used for DNA quantification were also used for this test. Beforehand, diethanolamine $1 \mathrm{M}(\mathrm{pH} 9.8)$ and STOP solutions (2 M $\mathrm{NaOH}$ and $0.2 \mathrm{mM}$ EDTA in distilled water) were prepared. Afterwards $20 \mu \mathrm{L}$ of the supernatant of each sample was inserted into 96-well plates, and then $60 \mu \mathrm{L}$ pNPP $2 \%$ solution (diluted in diethanolamine $1 \mathrm{M}$ ) was added, and incubated at $37^{\circ} \mathrm{C}$, for 45 minutes. Subsequently, $80 \mu \mathrm{L}$ STOP solution was added, and absorbance determined at $405 \mathrm{~nm}$. A standard curve was constructed with values between 2.0 and $0.25 \mathrm{mmol} / \mathrm{mL}$, for interpolation of the data and conversion into concentration values. After this, the values were normalized by the median of the control group and transformed into percentages.

\subsubsection{Calcium quantification}

Calcium quantification was determined using the Calcium Kit (Roche Diagnostics GmbH, Mannheim, Germany) ( $n=$ 8/group). In brief, at each pre-determined time point, the samples were washed in PBS, immersed in $800 \mu \mathrm{L}$ of $6 \mathrm{M}$ $\mathrm{HCl}$ and maintained at $-80^{\circ} \mathrm{C}$ for $24 \mathrm{~h}$. Subsequently, after defrosting at room temperature, $10 \mu \mathrm{L}$ of the supernatant of each sample was transferred to 96 -well plates. Then $70 \mu \mathrm{L}$ of solution R1 of the kit was added, and maintained at room temperature for 5 minutes. Afterwards, $160 \mu \mathrm{L}$ of solution $\mathrm{R} 2$ was added into the wells, and the plates were incubated at $37^{\circ} \mathrm{C}$ for 5 additional minutes. Measurements were taken in triplicate and absorbance was determined at $570 \mathrm{~nm}$. The data obtained by means of absorbance were interpolated into a calibration curve and then normalized by the mean of the control group and transformed into percentage values.

\subsubsection{Analysis of gene expression}

The expression of osteogenesis-related genes, namely alkaline phosphatase $(A L P L)$, runt-related transcription factor 2 (RUNX2) and collagen type I A 1 strand (COLIA1) was evaluated by relative real time polymerase chain reaction (RT-PCR) ( $n=5$ /group). Total RNA was extracted with Trizol (Invitrogen, Carlsbad, CA, USA) in accordance with the manufacturer's indications. The RNA concentration was determined by using a NanoDrop (NanoDrop ND -1000 Spectrophotometer, Alfagene, USA), and cDNA was synthesized from $10 \mathrm{ng}$ of RNA template by using the qScript $^{\mathrm{TM}}$ cDNA SuperMix kit (Quanta BioSciences, Gaithersbur, USA). The RT-PCR reaction was performed in a RT-PCR-Mastercycler (RealPlex, Eppendorf) using the qScript $^{\mathrm{TM}}$ One-Step qRT-PCR Kit (Quanta BioSciences, Gaithersbur, USA) and the primers listed in Table 1. The data was analyzed by the $\Delta \Delta \mathrm{Ct}$ method, comparing the experimental group with the control in each culture time interval evaluated and normalized by $18 \mathrm{~s}$ ribosomal (18S) endogenous control expression at day 0 . The data was transformed into fold change and comparative analysis was performed.

\subsection{Evaluation of platelet lysate-laden hyaluronic acid hydrogels chemotactic properties}

To study the chemotactic effect of HAPL hydrogels, hDPCs (at passage 7) were seeded in permeable PET translucent transwells (Corning, Durham, USA) $\left(5 \times 10^{4}\right.$ cells $/ \mathrm{cm}^{2}$ ) and incubated in culture medium for $24 \mathrm{~h}$ until forming a uniform monolayer. Then, cylindrical-shaped acrylic molds ( $5 \mathrm{~mm}$ diameter $\times 5 \mathrm{~mm}$ thick), 4 for test group/time point, were overlaid to the cell monolayer and, after injection of the HA/PL or HA solutions, the hydrogels were photocrosslinked, and cultured for up to 14 days. At each time-point, 1, 3, 7, and 14 days, the samples were stained with DAPI/Phalloidin and analyzed by confocal microscopy (TCS SP8, Leica, Germany) to evaluate the hDPCs inward migration into the hydrogels. 
Table 2 Flow cytometry analysis of different cell populations isolated from dental pulp tissue $(N=3)$. The numbers indicate the percentage of cells expressing a particular marker

\begin{tabular}{lllll}
\hline Marker (\%) & \multicolumn{2}{l}{ donor/hDPC sample } & Average \pm St. dev \\
\cline { 2 - 4 } & a & b & c & \\
\hline CD73 & 98.13 & 98.44 & 98.80 & $98.46 \pm 0.34$ \\
CD90 & 99.79 & 92.24 & 97.97 & $96.67 \pm 3.94$ \\
CD105 & 97.51 & 91.15 & 99.72 & $96.13 \pm 4.45$ \\
CD34 & 0.79 & 0.80 & 0.31 & $0.63 \pm 0.28$ \\
CD45 & 1.02 & 0.13 & 0.26 & $0.47 \pm 0.48$ \\
CD44 & 99.57 & 97.94 & 98.22 & $98.58 \pm 0.87$ \\
\hline
\end{tabular}

\subsection{Statistical analysis}

Statistical analysis of cell metabolism, DNA quantification, ALPL activity and calcium quantification was performed by Kruskal-Wallis and Mann-Whitney tests. Data from gene expression assay presented normal distribution and homoscedasticity, being therefore analyzed by the independent $\mathrm{t}$ test. The level of significance was set on $5 \%$.

\section{Results}

\subsection{Characterization of hDPCs population by flow cytometry}

Populations of hDPCs isolated from sound teeth were characterized using flow cytometry analysis at third passage to determine their phenotype. The results (Fig. 2b, Table 2) showed that the hDPCs populations are positive for mesenchymal stem cell markers such as CD73, CD90, CD105 and CD44 with over 96\% of cells expressing these markers and negative for the hematopoietic markers CD34 and CD45 $(<1 \%)$.

\subsection{Cell viability and proliferation}

The effect of PL incorporation into photocrosslinkable HA hydrogels over hDPCs was studied through the analysis of the cell viability (Fig. 3a), and proliferation (Fig. 3b). Overall, the hDPCs remained viable, with constant metabolic levels per cell, throughout the experiment for samples containing PL. In contrast, the formulations without PL presented a constant increase in the metabolic activity per cell $(p<0.05)$, which was significantly higher than the HAPL group $(p<0.05)$ after 21 days in culture. Conversely, the cell number in the hydrogels, herein measured as a function of dsDNA content (Fig. 3b), increased in the hydrogels containing PL while no differences were observed in control group throughout the 21 days' length of the assay $(p<0.05)$. Furthermore, the PL-laden hydrogels presented much higher hDPCs content for all the time points than the control group $(p<0.05)$.

\subsection{Alkaline phosphatase activity}

Alkaline phosphatase (ALPL) activity (Fig. 4a) in hDPCs cultured in osteogenic conditions was elevated throughout the time in culture, with its peak activity occurring at 21 days. Remarkably, the ALPL activity was consistently enhanced in the PL-laden group, when compared with the control, over time $(p<0.05)$.

\subsection{Calcium quantification}

Calcium deposition by hDPCs increased over time, reaching a maximum calcium concentration after 21 days of culture in osteogenic conditions. The hDPCs seeded over HAPL hydrogels showed higher $\mathrm{Ca}^{2+}$ deposition than the HA control group $(p<0.05)$, only after 21 days in culture (Fig. $4 b)$.

\subsection{Gene expression}

Analysis of the expression of genes that codify for alkaline phosphatase $(A L P L)$, collagen type I alpha 1 chain (COLIA1) and RUNX2 in hDPCs seeded over PL-laden (HAPL) or plain hydrogels (HA) after 7, 14, and 21 days of culture in osteogenic conditions in presented in Fig. 5. The expression of the gene coding for the transcription factor RUNX2; was higher in the initial time points, 7 and 14 days, in the HA control group $(p<0.05)$, while no significant differences were detected on day 21 (Fig. 5a). Conversely, statistical differences were detected $(p<0.05)$ for ALPL (Fig. 5b) and COLIAl (Fig. 5c) after 21 days in culture, which had higher expression in the HAPL group.

\subsection{Evaluation of PL chemotactic properties}

The analysis of DAPI/Phaloidin stained hydrogels photocrosslinked over hDPCs monolayers revealed that the $\mathrm{hDPCs}$ are resilient to the crosslinking reaction. Moreover, both HA and HAPL hydrogels were shown to be able to recruit hDPCs and support their adhesion, as soon as 3 days after the overlaying, and support cell proliferation, as depicted in Fig. 6. Nevertheless, the inward cell infiltration was limited to the first $20 \mu \mathrm{m}$ layer of the hydrogels, regardless the formulation.

\section{Discussion}

Recent approaches for the treatment of pulp injuries include the use of biomaterials [30], entangled with autogenous or 
Fig. 3 a) Metabolic activity per cell and b) DNA quantification at increasing culture times $(7$,

14 , and 21 days), $n=16$. Capital "A" indicate statistical difference with day 7

(Mann-Whitney test, $p<0.05$ ). Statistic differences between control (HA) and test (HAPL) groups within the same time point are indicated by an asterisk (Mann-Whitney test, $p<0.05$ )

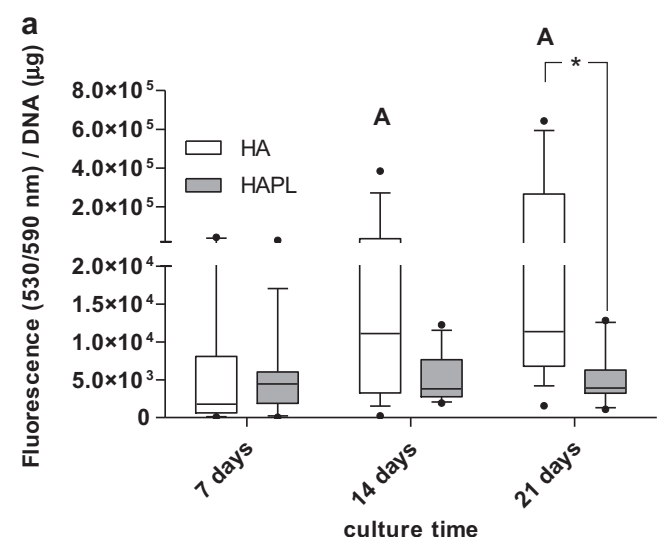

b

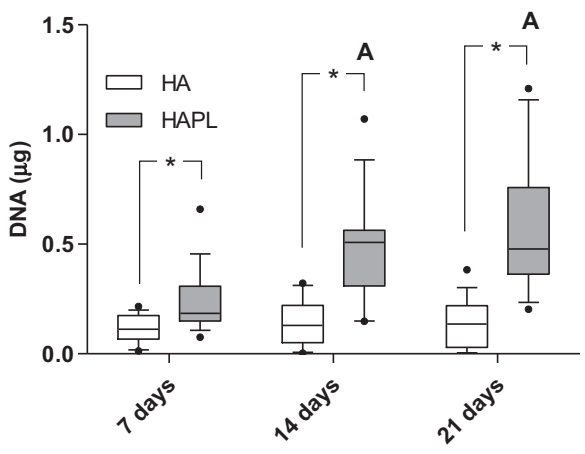

culture time

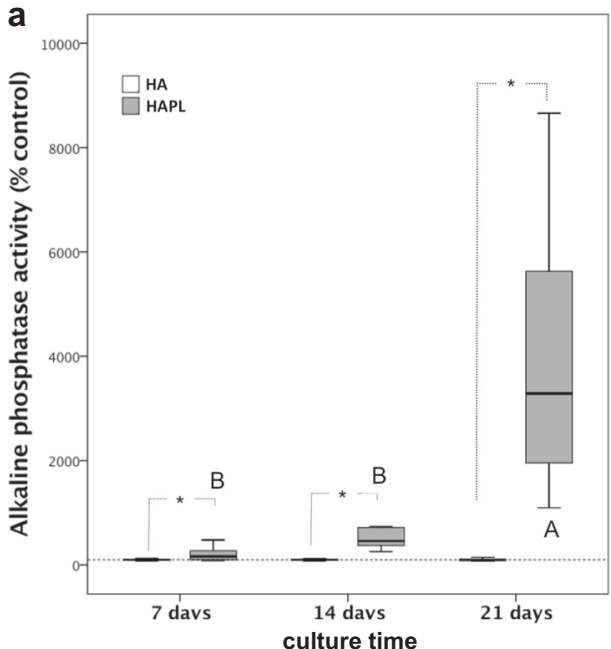

Fig. 4 Biochemical characterization of the cell response. a alkaline phosphatase activity and (b) calcium deposition by hDPCs after 7, 14, and 21 days of culture over HA and HAPL hydrogels in osteogenic conditions The values were normalize to the control group (HA) average, $n=8$. Different capital letters indicate statistical difference

heterogeneous growth factors. These signaling molecules may facilitate the tissue repair by controlling the endogenous wound healing mechanisms $[31,32]$. Thus, a microenvironment that favors host cell recruitment and differentiation is desirable [29]. The present study evaluated the effect of the association between PL and hyaluronic acid in protocrosslinkable hydrogels, on human dental pulp cells (hDPCs) cultures.

PL is a platelet rich hemoderivative (PRHd) obtained by disruption of the platelets contained in platelet concentrate (PC) or platelet rich plasma (PRP) by freeze/thaw cycles [21, 22] or ultrasounds [33]. Despite the consensual benefits of blood clot [34] or PRHds gels such as platelet rich fibrin (PRF) [35] to promote apex closure of immature teeth subjected to endodontic treatment and dentine-pulp complex regeneration, investigations regarding the application

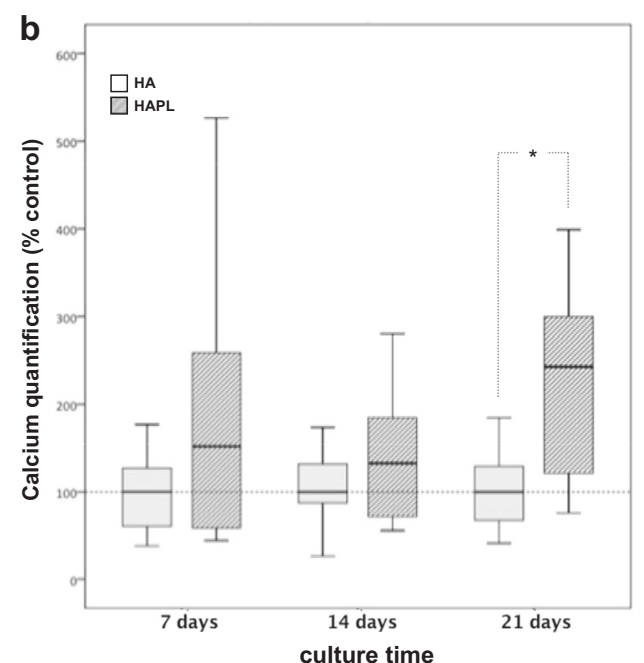

between time intervals within the experimental group (Mann-Whitney test, $p<0.05)$. Statistic differences between control (HA) and test (HAPL) groups within the same time point are indicated by an asterisk (Mann-Whitney test, $p<0.05$ )

of PL in the dentistry area are still incipient, and, to our knowledge, it was never attempted for endodontic regenerative treatments. The incorporation of PL in a system that could protect the GFs from degradation and allow their controlled release is expected to render more effective results. We have previously demonstrated that PL incorporation into photocrosslinkable HA hydrogels allowed the sustained release of GFs and enhanced the viability of cells encapsulated in- [18, 19] or seeded on- the hydrogels [18]. Moreover, HA hydrogels incorporating PL display bacteriostatic properties [18] which might be a real asset of the proposed system, given the potential of recurrent infections after endodontic procedures. In addition, these systems can also be frozen, stored and photocrosslinked after thawing, preserving the overall physicochemical and biological properties [19]. Furthermore, these hydrogels can be 
a
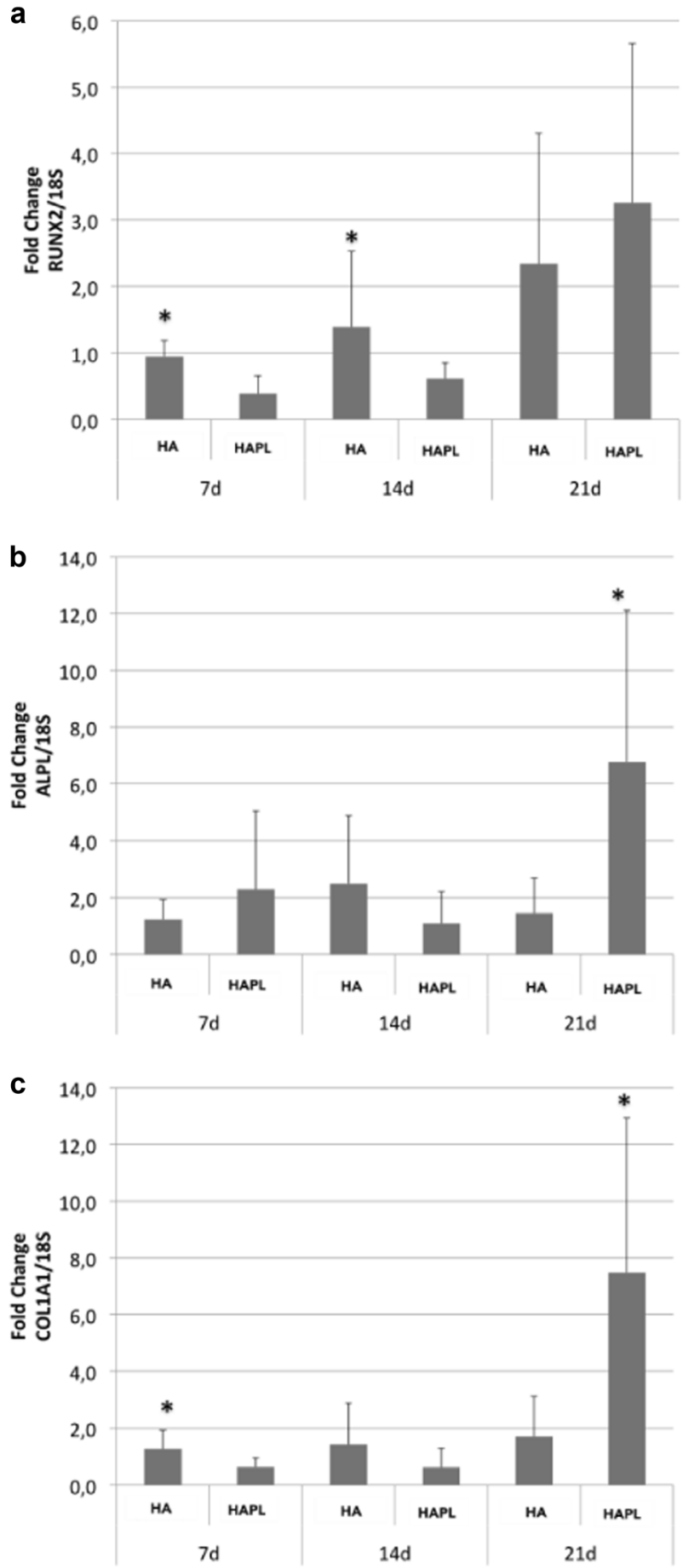

Fig. 5 Relative gene expression of (a) runt-related transcription factor 2 (RUNX2), (b) alkaline phosphatase (ALPL), and (c) collagen I alpha 1 chain (COLIA1) in human dental pulp cells seeded over experimental group (HAPL) and control (HA) hydrogels and cultured for 7 , 14 , and 21 days in osteogenic conditions. The gene expression was normalized using the $\Delta \Delta \mathrm{Ct}$ method by the expression of the endogenous control 18SRNA. Comparisons were made between the experimental and control groups in each evaluation period. Columns represent means and error bars represent the standard deviation, $n=5$ ). Asterisk (*) indicate statistically significant differences within the same time point (independent $t$ test, $p>0.05$ ) a

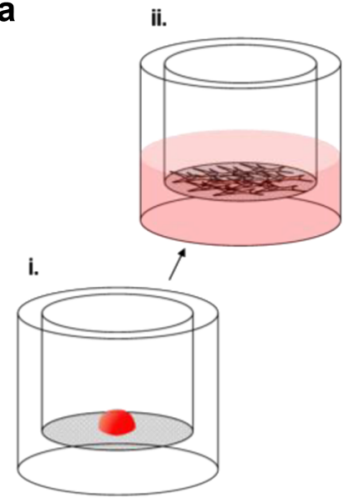

b
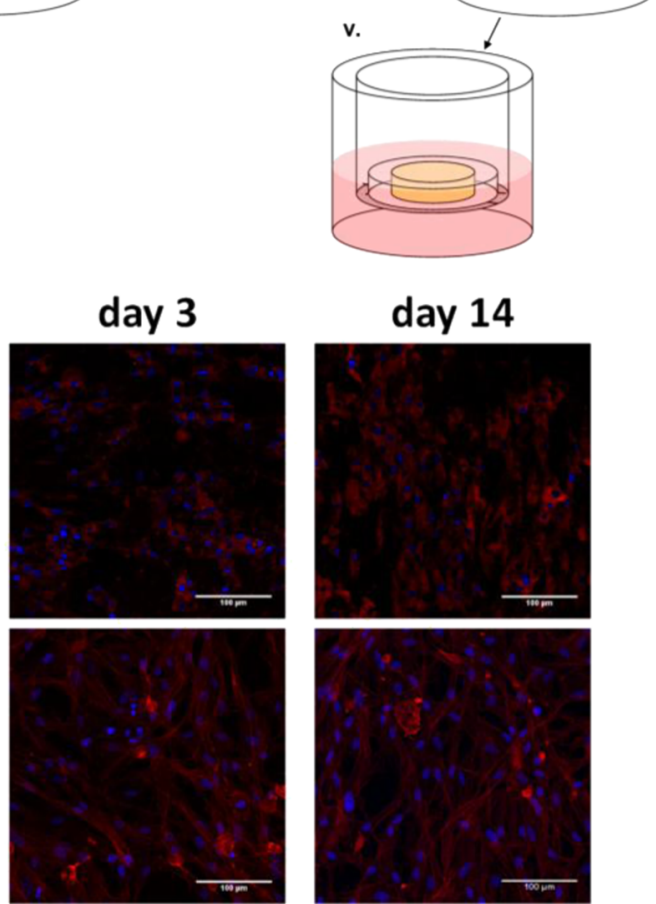

day 14

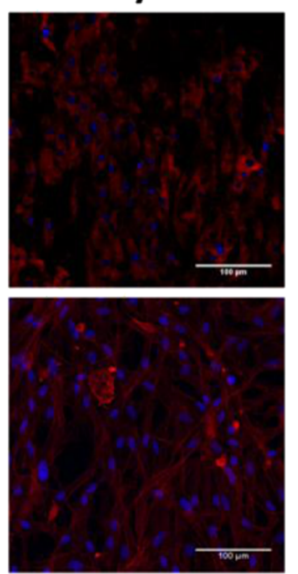

Fig. $6 \mathrm{hDPCs}$ response to hydrogels overlaying. a Assay scheme: (i) hDPCs were seeded on the surface of an adherent transwell and (ii) cultured until confluence; (iii) an acrylic mold was overlaid to the hDPCs layer, (iv) the Met-HA gel was injected and photocrosslinked, and (v) the hDPCs were co-cultured with the overlaid HA hydrogels for up to 14 days. b Representative confocal micrographs of DAPI/ Phaloidin stained hDPCs which migrated upward from a cell monolayer to HA or HAPL hydrogels photocrosslinked over them after 3 and 14 days of culture in basal medium

photocrosslinked by the camphorquinone (CQ)/tertiary amines photoinitiator system triggered by light curing systems already available in clinical practice. Therefore, the proposed system can be an off-the-shelf therapeutic for clinical applications.

Over $96 \%$ of plastic-adherent cells isolated from dental pulp under the scope of this study expressed the mesenchymal cell markers CD73, CD90, CD105 and CD44 and were negative for $\mathrm{CD} 34$ and $\mathrm{CD} 45$, as previously reported $[29,36]$. The hDPCs are able to produce mineralized nodules in vitro and form dentine-like tissue ectopically 
in vivo [29]. Therefore, they have been selected for in vitro assessment of TE approaches aiming endodontic regeneration. Within this study a positive effect in the association between PL and hyaluronic acid was found over hDPCs viability and proliferation. The high energy light source used for the photocrosslinking, associated with temperature increase, produce reactive oxygen species [37]. The scavenging mechanisms for ROS in eukaryotes are NADPH-dependent therefore affect the cellular metabolic activity [37], as herein reported (Fig. 3a). The incorporation of PL had a protective effect, as observed by the maintenance of the cell metabolic activity at basal levels in the HAPL group, as well as enhanced hDPCs proliferation. The mitogenic properties of PL $[22,38]$ and PL-incorporating hydrogels is well known $[18,19,39]$. In previous works, we reported the enhanced viability of periodontal ligament fibroblasts [18], adipose tissue-derived stem cells [19], or even umbilical cord endothelial cells [39] in hydrogels loaded with PL. It has been attributed to the release of PDGF, a growth factor reported to stimulate the proliferation of mesenchymal cells [40]. Furthermore, the fibrin present in PL $[41,42]$ would provide extra docking sites for cells to adhere and proliferate, as reported for other fibrin/ HA hydrogels reported in literature [43, 44].

The production of ALPL determines an initial event in the mineralization process [45]. Thus, the increased production of this protein is essential for the dentin-pulp complex reparative process, favoring the formation of tertiary dentin. Therefore, the effect of PL-laden hydrogels over the ALPL production by hDPCs was evaluated. In the present study, the primary hDPCs cultures displayed higher ALPL production in the presence of PL right from the initial stage of cultivation (7 days). It is necessary to emphasize that the ALPL activity depicted in Fig. 4a is normalized by the ALPL activity in the control group for each culture time in order to allow a direct inference of the PL effect. Therefore, despite produced in the control group, differences were observed between the control and experimental groups for all the evaluation times, with the peak of ALPL production being observed at 21 days for the samples cultivated on the hydrogels enriched with PL.

The analysis of $A L P L$ expression was coherent with the reported ALPL activity, being increased in the hDPCs seeded over the PL-laden hydrogels. The enhancement of the early osteogenic marker $A L P L$ expression of hDPCs cultured over PL-laden hydrogels was followed by the higher deposition of mineralized nodules and the increased expression of COLIA1 which composes the main protein constituent of bone ECM. With regards to the calcium deposition it is important to highlight that cells were obtained from different cell donors, and these cells can react differently to the stimulus, justifying the data variability.
The over-expression of RUNX2 by mesenchymal stem cells cultured in osteogenic differentiation medium, as herein observed for the hDPCs seeded over the HA or HAPL groups, is usually considered an early marker of osteogenesis. However, the RUNX2 gene in also a cell cycle regulator mediating the entry and exit of the $G 2 / M$ phases, with maximum of expression during G2 phase [37]. Since the hDPCs on HA hydrogels are not proliferating, the $R U N X 2$ higher expression for day 7 and 14 might be the combined effect of the mitosis arrest and the osteogenic induction. In fact, contrarily to what is observed in mice bone marrow stem cells (BMSCs) [46], the activation of osteoblastic differentiation of human BMSCs is not dependent on the overexpression of RUNX2 [47], as observed in HAPL hydrogels. Even maintaining a constitutive expression in osteogenic conditions, the activity of $R U N X 2$ can be modulated by post-transcriptional modifications, namely, by the phosphorylation of key residues [47], via several pathways, namely the mitogen-activated protein kinase (MAPK) [48] or Smad [48] signaling pathways.

The real osteogenic effect of PRHds, has been thoroughly debated in literature as the clinical outcomes of bone regenerative therapies using PRHds are very ambiguous. In fact, it seems that the positive effect of morphogenic GFs present in PL namely bFGF, TGF- $\beta 1$, TGF- $\beta 2$, and IGF [21, 22], which are known to stimulate the formation of tertiary dentin and dental pulp repair [24], may be overshadowed by the action of anti-osteogenic PDGF-BB [49]. Human mesenchymal stem cells derived from bone marrow [22] or hDPCs [36] maintain the multipotency after long-term culture in PL-supplemented media. However, PRHds have an additive or even synergistic effect when combined with osteoinductive materials [50,51], or, in the case of this study, osteogenic medium [36]. The incorporation of PL into HA hydrogels can, furthermore, modulate the microenvironmental enrichment with specific growth factors. For instance, Oliveira and co-workers showed that low sulfated polysaccharides, such as HA, presented high affinity to PDGF, while heparin showed high affinity for VEGF [52].

Of particular interest was the response of hDPCs monolayers to the hydrogel precursors and photocrosslinking reaction. These adhered to the superposed HA hydrogels, regardless the formulation, and started to colonize the hydrogels contact surface as soon as after 3 days in cultured and was visible, at day 14 , a confluent $20 \mu \mathrm{m}$ cell layer within the hydrogel. The biocompatibility of HA oligomers [53] or HA-based sponges [4] with amputated pulp tissue was already known from pre-clinical models in rats. Nevertheless, the anticipated chemotactic effect of PL [18, 54] was not verified. Most likely the dense polymeric mesh of the hydrogels impaired the inward migration of the cells. Future developments in this photocrosslinkable injectable 
system should procure to tune the hydrogel mesh density, namely by reducing the precursors density, or the crosslinking density, e.g., using Met-HA with lower modification degree. Nevertheless, this assay layout, roughly mimicking the amputated pulp inside a pulp chamber, give good insights for the applicability of these hydrogels in endodontic treatments. Therefore, the effect of PL conjugated with an injectable HA hydrogel, can be further investigated in a pre-clinical pulp amputation model.

\section{Conclusion}

Hyaluronic acid hydrogels incorporating platelet lysate protect cell viability and proliferation. Moreover, the incorporation of platelet lysate into HA hydrogels stimulate the expression of osteogenic markers and deposition of mineralized matrix by primary cultures of hDPCs. Additionally, the photocrosslinking reaction for hydrogel production is well tolerated by the hDPCs. Overall, the results herein reported provide strong evidences for the applicability of the proposed photocrosslinkable hydrogels incorporating PL for endodontic regenerative therapeutics.

Acknowledgements LFDA acknowledges Fundação de Amparo à Pesquisa do Estado de São Paulo (FAPESP) for the grant 2014/ 12017-8. Portuguese Foundation for Science and Technology (FCT) for PSB PhD grant SFRH/BD/73403/2010, MTR post-doctoral grant (SFRH/BPD/111729/2015), MEG grant (IF/00685/2012), and RECOGNIZE project (UTAP-ICDT/CTM-BIO/0023/2014), RL3TECT - NORTE-07-0124-FEDER-000020 project co-financed by ON.2 (NSRF) through ERD. This study also received financial support from FCT/Ministério da Ciência, Tecnologia, e Ensino Superior (FCT/MCTES) and Fundo Social Europeu through Programa Operacional do Capital Humano (FSE/POCH) PD/59/2013 for the LA ICVS-3Bs (UID/Multi/50026/2013). The authors would like to thank Maurizio Gulino, for its support in the in vitro experiments and Maló Clinic, Porto, Dra Ana Ferro and Dr Bruno Queridinha for the donation of permanent teeth.

\section{Compliance with ethical standards}

Conflict of interest The authors declare that they have no conflict of interest.

\section{References}

1. Silva CR, Gomez-Florit M, Babo PS, Reis RL, Gomes ME. 3D Functional scaffolds for dental tissue engineering. Funct. 3D Tissue Eng. Scaffolds. Elsevier; 2018. pp. 423-50.

2. Ferroni L, Gardin C, Sivolella S, Brunello G, Berengo M, Piattelli A, et al. A hyaluronan-based scaffold for the in vitro construction of dental pulp-like tissue. Int J Mol Sci. 2015;16:4666-81.

3. Chrepa V, Austah O, Diogenes A. Evaluation of a commercially available hyaluronic acid hydrogel (restylane) as injectable scaffold for dental pulp regeneration: an in vitro evaluation. J Endod. 2017;43:257-62.
4. Inuyama $\mathrm{Y}$, Kitamura $\mathrm{C}$, Nishihara $\mathrm{T}$, Morotomi T, Nagayoshi M, Tabata Y, et al. Effects of hyaluronic acid sponge as a scaffold on odontoblastic cell line and amputated dental pulp. J Biomed Mater Res Part B Appl Biomater. 2010;92B:120-8.

5. Soares DG, Rosseto HL, Basso FG, Scheffel DS, Hebling J, Costa $\mathrm{CA}$, de S. Chitosan-collagen biomembrane embedded with calcium-aluminate enhances dentinogenic potential of pulp cells. Braz Oral Res. 2016;30:1-10.

6. Dobie K, Smith G, Sloan AJ, Smith AJ. Effects of alginate hydrogels and TGF- $\beta 1$ on human dental pulp repair in vitro. Connect Tissue Res. 2002;43:387-90.

7. Kitamura C, Nishihara T,Terashita M,Tabata Y,Washio A. Local regeneration of dentin-pulp complex using controlled release of FGF-2 and naturally derived sponge-like scaffolds. Int J Dent. 2012;2012:1-8.

8. Ishimatsu H, Kitamura C, Morotomi T, Tabata Y, Nishihara T, Chen KK, et al. Formation of dentinal bridge on surface of regenerated dental pulp in dentin defects by controlled release of fibroblast growth factor-2 from gelatin hydrogels. J Endod. 2009;35:858-65.

9. Qu T, Jing J, Ren Y, Ma C, Feng JQ, Yu Q, et al. Complete pulpodentin complex regeneration by modulating the stiffness of biomimetic matrix. Acta Biomater. 2015;16:60-70.

10. Piva E, Silva AF, Nör JE. Functionalized scaffolds to control dental pulp stem cell fate. J Endod. 2014;40:S33-40.

11. Suzuki T, Lee CH, Chen M, Zhao W, Fu SY, Qi JJ, et al. Induced migration of dental pulp stem cells for in vivo pulp regeneration. J Dent Res. 2011;90:1013-8.

12. Galler KM, Cavender AC, Koeklue U, Suggs LJ, Schmalz G, D'Souza RN. Bioengineering of dental stem cells in a PEGylated fibrin gel. Regen Med. 2011;6:191-200.

13. Yang JW, Zhang YF, Sun ZY, Song GT, Chen Z. Dental pulp tissue engineering with bFGF-incorporated silk fibroin scaffolds. J Biomater Appl. 2015;30:221-9.

14. Jih G,Wu H,Chen M, Chen M, Chang S, Wang T. Control of three-dimensional substrate stiffness to manipulate mesenchymal stem cell fate toward neuronal or glial lineages. Acta Biomaterialia. 2013;9:5170-80.

15. Shibata S, Yoneda S, Yanagishita M, Yamashita Y. Isolation of proteoglycan (versican) aggregate from rat dental pulp. Arch Oral Biol. 2000;45:563-8.

16. Babo PS, Reis RL, Gomes ME. Production and characterization of hyaluronic acid microparticles for the controlled delivery of growth factors using a spray/dehydration method. J Biomater Appl. 2016;31:693-707.

17. Domingues RMA, Silva M, Gershovich P, Betta S, Babo P, Caridade SG, et al. Development of injectable hyaluronic acid/ cellulose nanocrystals bionanocomposite hydrogels for tissue engineering applications. Bioconjug Chem. 2015;26:1571-81.

18. Babo PS, Pires RL, Santos L, Franco A, Rodrigues F, Leonor I, et al. Platelet lysate-loaded photocrosslinkable hyaluronic acid hydrogels for periodontal endogenous regenerative technology. ACS Biomater Sci Eng. 2017;3:1359-69.

19. Neves LS, Babo PS, Gonalves AI, Costa-Almeida R, Caridade SG, Mano JF, et al. Injectable hyaluronic acid hydrogels enriched with platelet lysate as a cryostable off-the-shelf system for cellbased therapies. Regen Eng Transl Med. 2017;3:53-69. https:// doi.org/10.1007/s40883-017-0029-8.

20. Burdick JA, Chung C, Jia X, Randolph MA, Langer R. Controlled degradation and mechanical behavior of photopolymerized hyaluronic acid networks. Biomacromolecules. 2005;6:386-91.

21. Fekete N, Gadelorge M, Fürst D, Maurer C, Dausend J, FleuryCappellesso $\mathrm{S}$, et al. Platelet lysate from whole blood-derived pooled platelet concentrates and apheresis-derived platelet concentrates for the isolation and expansion of human bone marrow 
mesenchymal stromal cells: production process, content and identification of active comp. Cytotherapy. 2012;14:540-54.

22. Crespo-Diaz R, Behfar A, Butler GW, Padley DJ, Sarr MG, Bartunek J, et al. Platelet lysate consisting of a natural repair proteome supports human mesenchymal stem cell proliferation and chromosomal stability. Cell Transplant. 2011;20:797-812.

23. Zimmermann R, Jakubietz R, Jakubietz M, Strasser E, Schlegel A, Wiltfang J, et al. Different preparation methods to obtain platelet components as a source of growth factors for local application. Transfus. 2001;41:1217-24.

24. Tziafas D. Basic mechanisms of cytodifferentiation and dentinogenesis during dental pulp repair. Int J Dev Biol. 2017;39:281-90.

25. Chase LG, Lakshmipathy U, Solchaga LA, Rao MS, Vemuri MC. A novel serum-free medium for the expansion of human mesenchymal stem cells. Stem Cell Res Ther. 2010;1:8.

26. Costa-Almeida R, Franco AR, Pesqueira T, Oliveira MB, Babo PS, Leonor IB, Mano JF, Reis RL, Gomes ME. The effects of platelet lysate patches on the activity of tendon-derived cells. Acta Biomaterialia. 2018;68:29-40.

27. Nör JE. Buonocore memorial lecture. Oper Dent. 2006;31:633-42.

28. Silva ED, Babo PS, Costa-Almeida R, Domingues RMA, Mendes $\mathrm{BB}, \mathrm{Paz} \mathrm{E}$, et al. Multifunctional magnetic-responsive hydrogels to engineer tendon-to-bone interface. Nanomed Nanotechnol Biol Med. 2017.

29. Gronthos S, Mankani M, Brahim J, Robey PG, Shi S. Postnatal human dental pulp stem cells (DPSCs) in vitro and invivo. Proc Natl Acad Sci. 2000;97:13625-30.

30. Galler KM, Buchalla W, Hiller KA, Federlin M, Eidt A, Schiefersteiner M, et al. Influence of root canal disinfectants on growth factor release from dentin. J Endod. 2015;41:363-8.

31. Mao JJ, Kim SG, Zhou J, Ye L, Cho S, Suzuki T, et al. Regenerative endodontics. Dent Clin North Am. 2012;56:639-49.

32. Woo S, Kim W, Lim H. Combination of mineral trioxide aggregate and platelet-rich fibrin promotes the odontoblastic differentiation and mineralization of human dental pulp cells via BMP/ Smad signaling pathway. Journal of Endodontics. 2016;42:82-8.

33. Fortunato TM, Beltrami C, Emanueli C, De Bank PA, Pula G. Platelet lysate gel and e ndothelial progenitors stimulate microvascular network formation in vitro: tissue engineering implications. Sci Rep. 2016;6:25326.

34. Banchs F, Trope M. Revascularization of immature permanent teeth with apical periodontitis: new treatment protocol? J Endod. 2004;30:196-200.

35. Shivashankar VY, Johns DA, Vidyanath S, Kumar MrR, Shivashankar VY. Platelet rich fibrin in the revitalization of tooth with necrotic pulp and open apex. J Conserv Dent. 2012;15:395.

36. Chen B, Sun H-H, Wang H-G, Kong H, Chen F-M, Yu Q. The effects of human platelet lysate on dental pulp stem cells derived from impacted human third molars. Biomaterials. 2012;33:5023-35.

37. Apel K, Hirt H. Reactive oxygen species: metabolism, oxidative stress, and signal transduction. Annu Rev Plant Biol. 2004;55:373-99.

38. Wu R, Yu Y, Yin Y, Zhang X-Y, Gao L, Chen F-M. Platelet lysate supports the in vitro expansion of human periodontal ligament stem cells for cytotherapeutic use. J Tissue Eng Regen Med. 2017;11:2261-75.
39. Santo VE, Babo P, Amador M, Correia C, Cunha B, Coutinho DF, et al. Engineering enriched microenvironments with gradients of platelet lysate in hydrogel fibers. Biomacromolecules. 2016;17:1985-97.

40. Altaie A. Use of platelet lysate for bone regeneration-are we ready for clinical translation? World J Stem Cells. 2016;8:47.

41. Copland IB, Garcia MA, Waller EK, Roback JD, Galipeau J. The effect of platelet lysate fibrinogen on the functionality of MSCs in immunotherapy. Biomaterials. 2013;34:7840-50.

42. Renn T-Y, Kao Y-H, Wang C-C, Burnouf T. Anti-inflammatory effects of platelet biomaterials in a macrophage cellular model. Vox Sang. 2015;109:138-47.

43. Snyder TN, Madhavan K, Intrator M, Dregalla RC, Park D. A fibrin/hyaluronic acid hydrogel for the delivery of mesenchymal stem cells and potential for articular cartilage repair. J Biol Eng. 2014;8:10.

44. Zhang Y, Heher P, Hilborn J, Redl H, Ossipov DA. Hyaluronic acid-fibrin interpenetrating double network hydrogel prepared in situ by orthogonal disulfide cross-linking reaction for biomedical applications. Acta Biomater. 2016;38:23-32.

45. Golub EE, Boesze-Battaglia K. The role of alkaline phosphatase in mineralization. Curr Opin Orthop. 2007;18:444-8.

46. Gaur T, Lengner CJ, Hovhannisyan H, Bhat RA, Bodine PVN, Komm BS, et al. Canonical WNT signaling promotes osteogenesis by directly stimulating Runx2 gene expression. J Biol Chem. 2005;280:33132-40.

47. Shui C, Spelsberg TC, Riggs BL, Khosla S. Changes in Runx2/ Cbfa1 expression and activity during osteoblastic differentiation of human bone marrow stromal cells. J Bone Miner Res. 2003;18:213-21.

48. Xiao G, Jiang D, Gopalakrishnan R, Franceschi RT. Fibroblast growth factor 2 induction of the osteocalcin gene requires MAPK activity and phosphorylation of the osteoblast transcription factor, Cbfa1/Runx2. J Biol Chem. 2002;277:36181-7.

49. Kubota K, Sakikawa C, Katsumata M, Nakamura T, Wakabayashi K. Platelet-derived growth factor BB secreted from osteoclasts acts as an osteoblastogenesis inhibitory factor. J Bone Miner Res. 2002;17:257-65.

50. Malhotra A, Pelletier MH, Yu Y, Walsh WR. Can platelet-rich plasma (PRP) improve bone healing? A comparison between the theory and experimental outcomes. Arch Orthop Trauma Surg. 2013;133:153-65.

51. Oryan A, Alidadi S, Moshiri A. Platelet-rich plasma for bone healing and regeneration. Expert Opin Biol Ther. 2016;16:213-32.

52. Oliveira SM, Santo VE, Gomes ME, Reis RL, Mano JF. Layerby-layer assembled cell instructive nanocoatings containing platelet lysate. Biomaterials. 2015;48:56-65.

53. Sasaki T, Kawamata-Kido H. Providing an environment for reparative dentine induction in amputated rat molar pulp by high molecular-weight hyaluronic acid. Arch Oral Biol. 1995;40:209-19.

54. Leotot J, Coquelin L, Bodivit G, Bierling P, Hernigou P, Rouard $\mathrm{H}$, et al. Platelet lysate coating on scaffolds directly and indirectly enhances cell migration, improving bone and blood vessel formation. Acta Biomater. 2013;9:6630-40. 